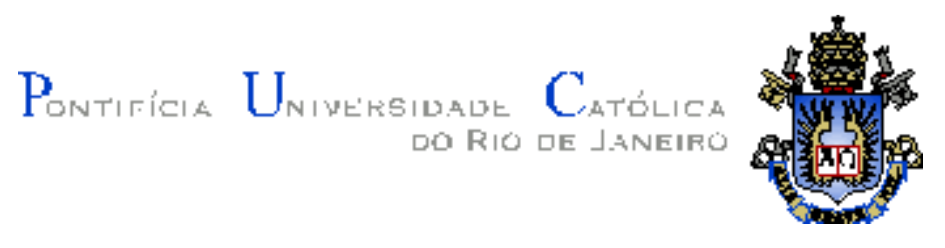

Márcio Jardim de Oliveira

\begin{abstract}
INDICAÇÃO DE DESCONTINUIDADES EM MATERIAIS METÁLICOS E COMPÓSITOS: UMA COMPARAÇÃO ENTRE MÉTODOS NÃO DESTRUTIV̧OS
\end{abstract}

Dissertação de Mestrado

Dissertação apresentada como requisito parcial para obtenção do grau de Mestre pelo Programa de PósGraduação em Engenharia de Materiais e de Processos Químicos e Metalúrgicos do Departamento de Engenharia de Materiais da PUCRio.

Orientador: Prof. Marcos Venicius Soares Pereira 


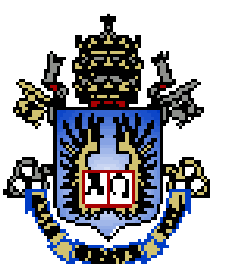

Márcio Jardim de Oliveira

\section{INDICAÇÃO DE DESCONTINUIDADES EM MATERIAIS METÁLICOS E COMPÓSITOS: UMA COMPARAÇÃO ENTRE MÉTODOS NÃO DESTRUTIVOS}

Dissertação apresentada como requisito parcial para obtenção do grau de Mestre pelo Programa de PósGraduação em Engenharia de Materiais e de Processos Químicos e Metalúrgicos do Departamento de Engenharia de Materiais da PUCRio. Aprovada pela Comissão Examinadora abaixo assinada.

Prof. Marcos Venicius Soares Pereira

Orientador

Departamento de Engenharia de Materiais - PUC-Rio

Prof. Fathi Ibrahim Darwish

Universidade Federal Fluminense

Prof. Felipe José da Silva

Instituto Federal de Educação, Ciência e Tecnologia do Rio de Janeiro

\section{Prof. Sérgio Damasceno Soarres \\ CENPES/Petrobrás}

Prof. José Eugenio Leal

Coordenador Setorial de Pós-Graduação do Centro Técnico Científico da

PUC-Rio

Rio de Janeiro, 10 de Abril de 2014. 
Todos os direitos reservados. É proibida a reprodução total ou parcial do trabalho sem autorização da universidade, do autor e do orientador.

\section{Márcio Jardim de Oliveira}

Graduou-se em engenharia mecânica na Fundação Técnico Educacional Souza Marques em 1996. Trabalhou como desenhista, projetista, supervisor e coordenador em indústrias mecânicas por mais de 20 anos. Pós-graduado em docência do ensino superior. É professor da disciplina de desenho técnico da FAETEC desde 1998. Coordenador do curso técnico de mecânica do IFRJ.

Ficha Catalográfica

Indicação de descontinuidades em materiais metálicos e compósitos: uma comparação entre métodos não destrutivos / Márcio Jardim de Oliveira ; orientador: Marcos Venicius Soares Pereira. - 2014.

140 f. : il. (color.) ; $30 \mathrm{~cm}$

$$
\text { Dissertação (mestrado)-Pontifícia }
$$

Universidade Católica do Rio de Janeiro, Departamento de Engenharia de Materiais, 2014.

Inclui bibliografia

1. Engenharia de materiais - Teses. 2. Ensaios de materiais. 3. Descontinuidades simuladas. 4. Radiação penetrante. 5. Partículas magnéticas. 6 . Ultrassom. I. Pereira, Marcos Venicius Soares. II. Pontifícia Universidade Católica do Rio de Janeiro. Departamento de Engenharia de Materiais. III. Título. 
A minha esposa Daysi e minha filha Débora. 


\section{Agradecimentos}

Somente quem escreve uma dissertação de mestrado, tese de doutorado ou até mesmo um livro, sabe o trabalho árduo e estafante que essa tarefa impõe. Quantas vezes a obrigação nos exige postergar um passeio, uma visita ou um dia de felicidade com quem amamos. Por isso, nesse momento agradeço a todos que com amor, amizade, carinho e apoio, de uma maneira ou outra contribuíram para realização desse trabalho.

Primeiramente a Deus, pois sem ele em nossas vidas nada seria possível.

Aos meus pais Mario e Zulimar, pois foram os alicerces dessa caminhada.

A todos os colaboradores da empresa Arctest principalmente ao Sr. Paulo Stampone e ao amigo Mario Moura por todo auxílio durante a realização dos ensaios não destrutivos.

Aos amigos André Rocha Pimenta, José Maria Paolucci e Roberto Rocco e pelo incentivo.

Ao professor Francisco Nipo do CEFET Itaguaí pela ajuda nos ensaios de ultrassom.

A todos os professores e funcionários do DEMa por todo apoio e conhecimento passado de forma motivadora.

Ao CAPES e a PUC-Rio, pelos auxílios concedidos, sem os quais este trabalho não poderia ter sido realizado.

A todos os companheiros de Mestrado em especial Gilvania Terto, Roberta Amorim e Alex Galiza por todas as alegrias e tristezas compartilhadas.

Ao Prof. Marcos Venicius Soares Pereira por sua amizade, orientação, paciência, apoio e principalmente por suas aulas inspiradoras de mecânica da fratura. 


\section{Resumo}

Oliveira, Márcio Jardim de Oliveira; Pereira, Marcos Venicius Soares (orientador). Indicação de descontinuidades em materiais metálicos e compósitos: uma comparação entre métodos não destrutivos. Rio de Janeiro, 2014. 140 p. Dissertação de Mestrado - Departamento de Engenharia de Materiais e Metalurgia, Pontifícia Universidade Católica do Rio de Janeiro.

O parque industrial nos países em desenvolvimento necessita, cada vez mais, se adaptar a uma nova filosofia de serviço em que equipamentos considerados como seguros são aqueles que podem ser inspecionadas de forma confiável. Neste contexto, ensaios não destrutivos (END) são valiosas ferramentas usadas no controle de qualidade, manutenção de componentes e, principalmente, na avaliação da integridade estrutural de tais equipamentos. Este trabalho teve como objetivo avaliar a eficiência de diferentes técnicas de END (radiação penetrante, partículas magnéticas e ultrassom) na detecção de descontinuidades simuladas em amostras de material metálico e de compósito. Os resultados experimentais mostraram que os ensaios com raios $\mathrm{X}$, dentre todas as técnicas adotadas na inspeção da amostra metálica, apresentou a maior eficiência na detecção das diferentes geometrias de descontinuidades. Entretanto, nenhuma das técnicas de END possibilitou a detecção de descontinuidades na amostra do material compósito.

\section{Palavras-chave}

Ensaios de materiais; descontinuidades simuladas; radiação penetrante; partículas magnéticas; ultrassom. 


\section{Abstract}

Oliveira, Márcio Jardim de; Pereira, Marcos Venicius Soares (Advisor); Pereira. Discontinuities indication in metallic and composite materials: a comparison of non-destructive methods. Rio de Janeiro, 2014. 140 p. MSc. Dissertation - Departamento de Engenharia de Materiais e Metalurgia, Pontifícia Universidade Católica do Rio de Janeiro.

The industrial park in developing countries need, increasingly, to adapt to a new service philosophy in which equipments considered as safe are those that can be inspected in a reliable way. In this context, non-destructive testing (NDT) are valuable tools used in quality control, maintenance of components and especially in assessing the structural integrity of such equipment. This study aimed to evaluate the efficiency of different NDT techniques (penetrating radiation, ultrasound and magnetic particles) in the detection of simulated discontinuities in samples of metallic and composite materials. The experimental results showed that the X-ray, considering all techniques adopted in the inspection of the metallic sample, showed the highest efficiency in the detection of different geometries of discontinuities. However, anynone of NDT techniques allowed the detection of discontinuities in the sample of the composite material.

\section{Keywords}

Materials testing; simulated discontinuities; penetrating radiation; magnetic particles; ultrasound. 


\section{Sumário}

1. INTRODUÇÃO 18

2. REVISÃO DE LITERATURA 20

2.1. Ensaios destrutivos 20

2.2. Ensaios não destrutivos 22

2.3. Ultrassom 29

2.3.1. Histórico 29

2.3.2. Princípios do método 30

2.3.3. Velocidade de propagação 32

2.3.4. Atenuação sônica 33

2.3.5. Impedância acústica 34

2.3.6. Comportamento do feixe sônico 35

2.3.7. Campo próximo 36

2.3.8. Campo distante 36

2.3.9. Propagação das ondas $\quad 37$

2.3.9.1. Ondas longitudinais 37

2.3.9.2. Ondas transversais 38

2.3.9.3. Ondas superficiais 39

2.3.9.4. Ondas Lamb 40

2.4. Equipamentos do ensaio 41

2.4.1. Cabeçotes 42

2.4.1.1. Cabeçote normal ou reto 43

2.4.1.2. Cabeçote angular 43

2.4.1.3. Cabeçote duplo cristal 44

2.5. Acoplantes 45

2.6. Técnicas de inspeção 45

2.6.1. Método de pulso-eco 46

$\begin{array}{ll}\text { 2.6.2. Método da transparência } & 47\end{array}$

$\begin{array}{ll}\text { 2.6.3 Método por imersão } & 47\end{array}$

2.7. Vantagens e desvantagens do ensaio por ultrassom 48 
2.8. Raios X 49

2.8.1. Histórico 49

2.9. Radioatividade natural e artificial 50

2.10. Fontes de radiação ionizante 54

2.11. Propriedade dos raios $X \quad 54$

2.12. Produção dos raios $X \quad 54$

2.13. Produção dos raios gama 56

2.14. Atividade de uma fonte radioativa 58

2.15. Meia vida $\quad 59$

2.16. Irradiadores 60

2.17. Detectores de radiação 61

2.18. Registro radiográfico 62

2.19. Método de trabalho 62

2.20 Indicadores de qualidade de imagem (IQI) 63

2.20.1. IQI de arame ou fio 64

2.20.2. IQI de chapa ou furo 65

2.21. Vantagens e desvantagens do ensaio por radiação penetrante 66

2.22. Ensaio por partículas magnéticas 66

2.22.1 Histórico 66

2.22.2. Principais grandezas $\quad 67$

2.22.2.1. Pólos magneticos $\quad 67$

2.22.2.2. Campo magnético 67

2.22.2.3. Fluxo magnético 68

2.22.2.4. Permeabilidade magnética 69

2.23. Classificação magnética dos materiais 69

2.24. Histerese magnética 70

2.25 Descrição da técnica $\quad 71$

2.26. Métodos de magnetização 72

2.26.1. Magnetização longitudinal 72

2.26.2. Magnetização circular 73

2.26.3 Magnetização multidirecional $\quad 74$

2.27. Técnicas de magnetização $\quad 75$

$\begin{array}{ll}\text { 2.27.1 Técnica do Yoke } & 76\end{array}$ 
2.28. Desmagnetização 77

2.29. Partículas magnéticas $\quad 77$

2.30. Vantagens e desvantagens do ensaio 78

3. MATERIAIS E MÉTODOS 80

3.1. Preparação das amostras 81

3.1.1. Amostra metálica 81

3.1.2. Amostra de compósito 86

3.2. Ensaios radiográficos 91

3.3. Ensaio radiográfico computadorizado 103

3.4. Equipamento radiográfico 103

3.5. Partículas magnéticas 104

$\begin{array}{ll}\text { 3.6. Aparelho } & 104\end{array}$

$\begin{array}{ll}\text { 3.7. Processos } & 105\end{array}$

$\begin{array}{ll}\text { 3.7.1. Via úmida visivel } & 105\end{array}$

$\begin{array}{ll}\text { 3.7.2. Via úmida fluorescente } & 107\end{array}$

$\begin{array}{ll}3.8 \text { Ensaio por ultrassom } & 109\end{array}$

4. RESULTADOS E DISCUSSÃO 112

$\begin{array}{ll}\text { 4.1. Ensaio radiográfico } & 115\end{array}$

4.2. Ensaio por partículas magnéticas $\quad 127$

$\begin{array}{ll}\text { 4.3. Ensaio por ultrassom } & 131\end{array}$

4.4. Comparação entre as técnicas adotadas 134

5.0. CONCLUSÕES 135

6.0. RECOMENDAÇÕES DE TRABALHOS FUTUROS 137

$\begin{array}{ll}\text { 7.0. REFERÊNCIAS BIBLIOGRÁFICAS } & 138\end{array}$ 


\section{Lista de figuras}

Figura 1 - Exemplos de corpos de prova usados nos ensaios destrutivos [3]

Figura 2 - Descontinuidades superficiais e subsuperficiais e seus

diferentes métodos de detecção. Adaptada de [10]

Figura 3 - Descontinuidades internas e seus diferentes métodos de detecção. Adaptada de [10]

Figura 4 - Inspeção externa não intrusiva de esfera. A - Montagem de andaimes pela parte externa da esfera; B - Utilização de inspetores escaladores na inspeção externa;

Figura 5 - Tipos de sons em função da frequência [22]

Figura 6 - Esquema de inspeção ultrassônica e gráfico.

Adaptada de [1]

Figura 7 - Princípio básico do método por ultrassom [22] 32

Figura 8 - Reflexão e transmissão da onda sônica [18] 35

Figura 9 - Áreas ou zonas do feixe sônico [22] 35

Figura 10 - Estruturas atômicas, gás, líquido e sólido [18]

Figura 11 - Esquema de propagação das ondas longitudinais.

Adaptada [24]

Figura 12 - Esquema de propagação das ondas de compressão [25]

Figura 13 - Esquema de propagação das ondas Rayleigh.

Adaptada de [24]

Figura 14 - Propagação das ondas Lamb. Adaptada de [24]

Figura 15 - Principais elementos de um cabeçote padrão [25]

Figura 16 - Esquema de um cabeçote normal ou reto [22]

Figura 17 - Esquema básico de um transdutor angular.

Adaptada de [24]

Figura 18 - Esquema básico de um cabeçote duplo cristal [22]

Figura 19 - Representação esquemática da técnica

ultrassônica de pulso-eco [22] 
Figura 20 - Representação esquemática da técnica ultrassônica por transparência [22] $\quad 47$

Figura 21 - Esquema da técnica ultrassônica por imersão [22] 48

Figura 22 - Radiografia da mão de Bertha esposa de Roentgen [27] 50

Figura 23 - Modelo atômico apresentado por Rutherford-Bohr.

Adaptada [29] 51

Figura 24 - Camadas eletrônicas e níveis de energia [7] 51

Figura 25 - Espectro magnético [31] 52

Figura 26 - Tubo de Colidge [30] 55

Figura 27 - Tubo gerador de raios X [33] 55

Figura 28 - Desenho esquemático representando a radioatvidade [35] 56

Figura 29 - Curva de decaimento do Irídio [25] 59

Figura 30 - Fonte selada radioativa [27] 60

Figura 31 - Foto de um irradiador 61

Figura 32 - Desenho esquemático de um irradiador em corte [25] 61

Figura 33 - Composição do filme radiográfico [30] 62

Figura 34 - Fundamentos do ensaio radiográfico [11] 63

Figura 35 - Posicionamento do IQI [30] 64

Figura 36 - IQI tipo fio (Norma ASTM) [25] 65

Figura 37 - IQI tipo furo (Norma ASTM) [30] 65

Figura 38 - Imã com pólos magnéticos norte e sul [42] 67

Figura 39 - Representação dos campo magnéticos e

as linhas de indução [42] 68

Figura 40 - Campo magnéticos longitudinal, circular e descontinuidades em diferentes angulações. Adaptada de [24] 69

Figura 41 - Curva de histerese magnética

$\mathrm{B}$ (densidade de fluxo) $\times \mathrm{H}$ (força magnetizante) 71

Figura 42 - Origem dos campos de fuga [42] 72

Figura 43 - Exemplo de componente sob ação da magnetização longitudinal. Adpatada de [24] 73

Figura 44 - Exemplo de um componente sob ação da magnetização circular. Adaptada de [24] $\quad 74$

Figura 45 - Esquema básico de um yoke. [25] 76 
Figura 46 - Esquema de um yoke em uso. Adaptada de [24] 77

Figura 47 - Tambor metálico $\quad 80$

Figura 48 - Tubo de compósito 81

Figura 49 - Esquema de retirada da amostra metálica 82

Figura 50 - Detalhamento das descontinuidades circulares amostra metálica 83

Figura 51 - Representação esquemática dos diâmetros

adotados nas furações das amostras 83

Figura 52 - Representação esquemática das espessuras residuais de parede (\%) após furação das amostras

Figura 53 - Detalhamento das descontinuidades lineares verticais na amostra metálica

Figura 54 - Detalhamento das descontinuidades lineares horizontais na amostra metálica

Figura 55 - Detalhamento das descontinuidades irregulares na amostra metálica

Figura 56 - Conjunto de descontinuidades usinadas por eletroerosão na amostra metálica

Figura 57 - Confecção de descontinudades com espátula 87

Figura 58 - Numeração de 1 a 3 nas descontinuidades 87

Figura 59 - Confecção das descontinuidades com ferro de solda

Figura 60 - Numeração de 4 a 6 nas descontinuidades 88

Figura 61 - Ferramentas utilizadas $\quad 89$

Figura 62 - Fluxograma ilustrando o procedimento experimental $\quad 90$

Figura 63 - Bunker usado nos ensaios radiográficos 91

Figura 64 - Irradiador adotado nos experimentos 93

Figura 65 - Comando a distância para liberação dos isótopos 93

Figura 66 - Equipamento usado nos ensaios por raios -X 94

Figura 67 - Filme radiográfico KODAK M100 usado no ensaio 95

Figura 68 - Etiqueta do filme usado 95

Figura 69 - Telas intensificadoras 96

Figura 70 - Etiqueta identificadora Arctest 97 
Figura 71 - Identificação do filme radiográfico

Figura 72 - Desenho esquemático da montagem do Chassi 98

Figura 73 - Chassi, tela intensificador e filme 98

Figura 74 - Posicionamento do IQI 94

Figura 75 - Distância fonte filme na amostra metálica 100

Figura 76 - Distância fonte filme no tubo de compósito 100

Figura 77 - Layout do laboratório 101

Figura 78 - Desenho esquemático do tanque com agentes químicos usados na revelação dos filmes radiográficos 102

Figura 79 - Secagem do filme na estufa 103

Figura 80 - Laboratório para execução ensaio radiográfico computadorizado 104

Figura 81 - Yoke usado no ensaio $\quad 105$

Figura 82 - Tinta de contraste usada no ensaio

por partículas magnéticas 106

Figura 83 - Tinta de constraste sendo usada na amostra metálica 106

Figura 84 - Execução do ensaio por partículas magnéticas 107

Figura 85 - Inspeção com as pernas do yoke na medida de $100 \mathrm{~mm} \quad 108$

Figura 86 - Inspeção com as pernas do yoke na medida de $30 \mathrm{~mm} \quad 108$

Figura 87 - Execução do ensaio por utrassom método pulso eco na amostra metálica 109

Figura 88 - Execução do ensaio por ultrassom método pulso eco na amostra do tubo compósito 110

Figura 89 - Calibração do aparelho de ultrassom 111

Figura 90 - Execução do ensaio por ultrassom pelo método medição de espessura na amostra do tubo compósito

Figura 91 - Representação esquemática das descontinuidades

simuladas na amostra metálica

Figura 92 - Representação esquematica das descontinuidades

simuladas na amostra do compósito

Figura 93 - Radiografia convencional com Selênio - 75

na amostra metálica

Figura 94 - Radiografia convencional com Selênio - 75 
na amostra metálica, após tratamento digital de imagem

Figura 95 - Radiografia computadorizada com Selênio - 75

na amostra metálica

Figura 96 - Radiografia computadorizada com Selênio - 75

Figura 97 - Radiografia convencional com Irídio - 192

na amostra metálica

Figura 98 - Radiografia convencional com Irídio - 192

na amostra metálica, após tratamento digital de imagem

Figura 99 - Radiografica computadorizada com Irídio - 192

na amostra metálica

Figura 100 - Radiografia computadorizada com Irídio - 192

na amostra metálica, após tratamento digital de imagem

Figura 101 - Radiografia convencional com raios $X$

na amostra metálica

Figura 102 - Radiografia convencional com raios $X$

na amostra metálica após tratamento digital de imagem

120

Figura 103 - Radiografia computadorizada com raios X na amostra metálica

Figura 104 - Radiografia computadorizada com raios $X$

na amostra metálica, após tratamento digital de imagem

Figura 105 - Radiografia convencional com Selênio - 75

na amostra do compósito

Figura 106 - Radiografia convencional com Selênio - 75

na amostra do compósito, após tratamento digital de imagem

Figura 107 - Radiografia digital com Selênio -75

na amostra do compósito

Figura 108 - Radiografia digital com Selênio - 75 na amostra do

compósito, após tratamento digital de imagem

Figura 109 - Radiografia convencional com Irídio - 192

na amostra do compósito

Figura 110 - Radiografia convencional com Irídio - 192

na amostra do compósito, após tratamento digital de imagem

Figura 111 - Radiografia digital com Irídio - 192 
na amostra do compósito

Figura 112 - Radiografia digital com Irídio - 192 na amostra

do compósito, após tratamento digital de imagem

Figura 113 - Radiografia convencional com raios X na amostra do compósito

Figura 114 - Radiografia convencional com raios X na amostra do compósito, após tratamento digital de imagem

Figura 115 - Radiografia digital com raios X na amostra do compósito 126 Figura 116 - Radiografia digital com raios X na amostra do compósito, após tratamento digital de imagem

Figura 117 - Detecção de descontinuidade limear (1).

Figura 118 - Detecção das descontinuidades circulares (8,9, 11 e 12) 128

Figura 119 - Detecção de descontinuidade irregular (3) 128

Figura 120 - Descontinuidades não detectadas no ensaio método via úmida colorida.

Figura 121 - Detecção de descontinuidade linear (1).

Figura 122 - Detecçção das descontinuidades circulares.

Figura 123 - Descontinuidades não detectadas no

ensaio por via úmida fluorescente.

Figura 124 - Tela do aparelho método pulso-eco na região sem descontinuidades da amostra metálica.

Figura 125 - Tela do aparelho método pulso eco na região com descontinuidades da amostra metálica.

Figura 126 - Medição da espessura da parede do tubo compósito 3,9 $\mathrm{mm}$.

Figura 127 - Medição da espessura da parede do tubo compósito $4,9 \mathrm{~mm}$. 


\section{Lista de Tabelas}

Tabela 1 - Métodos de ensaio x variáveis. Adaptada de [8] 24

Tabela 2 - Comparação entre ensaios não destrutivos.

Adaptada de [14] 28

Tabela 3 - Atenuação sônica de diferentes materiais [22]. 34

Tabela 4 - Diferenças entre transdutores ultrassônicos

Adaptada de [1] 42

Tabela 5 - Comparação entre os raios penetrantes $X$ e gama.

Adaptada de [31].

Tabela 6 - Principais radioisótopos usados nos raios gama $\quad 57$

Tabela 7 - Comparação entre variáveis que interferem na escolha das radiações penetrantes [35].

Tabela 8 - Vantagens e limitações das técnicas de magnetização [24]. 75

Tabela 9 - Grupo material 1.

Tabela 10 - Atividade dos isótopos usados nos ensaios radiográficos. 92

Tabela 11 - Modelos de irradiadores usados nos ensaios radiográficos. 92

Tabela 12 - Comparação entre tipos e classes de filmes. 94

Tabela 13 - Espessura das telas intensificadoras 96

Tabela 14 - Equivalência entre os IQl's. 99

Tabela 15 - Tempos de exposição das radiografias. 101

Tabela 16 - Agentes químicos usados na revelação dos filmes radiográficos. 102

Tabela 17 - Yoke usado no ensaio por partículas magnéticas; 105

Tabela 18 - Dimensões e profundidades das descontinuidades

na amostra metálica. 113

Tabela 19 - Dimensões e profundidades das descontinuidades

na amostra do compósito. 114

Tabela 20 - Detecção das descontinuidades na amostra metálica 134

Tabela 21 - Detecção das descontinidades na amostra do compósito 134 